\title{
Optical and Electrical Measurements Reveal the Orientation Mechanism of Homoleptic Iridium-Carbene Complexes
}

\author{
Markus Schmid, Kristoffer Harms, Carl Degitz, Thomas Morgenstern, Alexander Hofmann, \\ Pascal Friederich, Hans-Hermann Johannes, Wolfgang Wenzel, Wolfgang Kowalsky, \\ and Wolfgang Brütting*
}

Cite This: ACS Appl. Mater. Interfaces 2020, 12, 51709-51718

Read Online

ACCESS | Lill Metrics \& More | 回 Article Recommendations | st Supporting Information

ABSTRACT: Understanding and controlling the driving forces for molecular alignment in optoelectronic thin-film devices is of crucial importance for improving their performance. In this context, the preferential orientation of organometallic iridium complexes is in the focus of research to benefit from their improved light-outcoupling efficiencies in organic lightemitting diodes (OLEDs). Although there has been great progress concerning the orientation behavior for heteroleptic Ir complexes, the mechanism behind the alignment of homoleptic complexes is still unclear yet. In this work, we present a sky-blue phosphorescent dye that shows

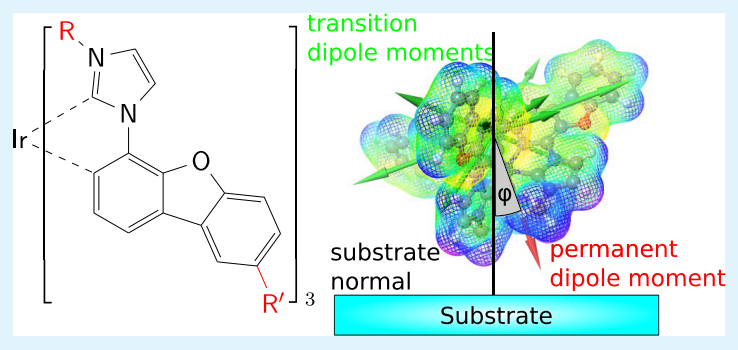
variable alignment depending on systematic modifications of the ligands bound to the central iridium atom. From an optical study of the transition dipole moment orientation and the electrically accessible alignment of the permanent dipole moment, we conclude that the film morphology is related to both the aspect ratio of the dye and the local electrostatic interaction of the ligands with the film surface during growth. These results indicate a potential strategy to actively control the orientation of iridium-based emitters for the application in OLEDs.

KEYWORDS: organic light-emitting diode, emitter orientation, orientation polarization, Ir complexes, meridional and facial isomers

\section{INTRODUCTION}

Understanding the orientation mechanisms of organic molecules is of crucial importance as it has a significant influence on many device parameters for organic light-emitting diodes (OLEDs), like charge carrier injection ${ }^{1-3}$ and transport ${ }^{4,5}$ as well as, especially, light outcoupling. ${ }^{6-12}$ Regarding the latter, the alignment of the emitting species has attracted considerable attention after its first observation with fluorescent and phosphorescent dyes in 2010 and 2011, respectively. ${ }^{6,7,13}$ The reason is that this feature offers a large potential to boost the OLED efficiency by increasing the amount of light extracted from the device by up to $50 \%{ }^{7,9,10,14}$ Exceptionally high efficiencies were achieved by exploiting this effect in the context of phosphorescent Ir-based dyes. ${ }^{13,15-17}$

There has been huge progress in explaining the orientation behavior for heteroleptic organometallic iridium complexes having two opto-electronically active ligands plus one ancillary ligand that is believed to drive their orientation upon evaporation on a suitable surface. Many of these emitters were investigated by angular-dependent photoluminescence spectroscopy (ADPL), revealing phenomenological design rules for a preferential alignment of their transition dipole moment (TDM). ${ }^{10,14}$ These rules could be reproduced by molecular-scale simulations of the thermal deposition process, giving further insight into the film morphology. ${ }^{18-20}$ Moreover, our group was recently able to show that the permanent dipole moment (PDM), which can be probed by impedance spectroscopy even at low dye concentrations relevant for device application, can provide further insights into molecular orientation. $^{21}$

In contrast to this remarkable progress for heteroleptic complexes, only a few studies were performed on homoleptic compounds having three identical opto-electronically active ligands. The main reason probably is that only a few of them were found to show alignment. ${ }^{10,14,22}$ Homoleptic compounds offer less degrees of freedom in their chemical design as the same ligand, abbreviated here symbolically as $\mathrm{C}^{\wedge} \mathrm{N}$ (although the formally more correct notation for the studied $\mathrm{N}$ heterocyclic carbene complexes is $\mathrm{C}^{\wedge} \mathrm{C}$ :), is octahedrally bound to the central Ir atom three times $\operatorname{Ir}\left(\mathrm{C}^{\wedge} \mathrm{N}\right)_{3}$. Nevertheless, depending on the binding of the ligands, two different isomers of these complexes have to be distinguished. Figure 1a shows a schematic representation of both possible configurations. While the so-called facial isomer exhibits a

Received: August 17, 2020

Accepted: October 20, 2020

Published: November 9, 2020 


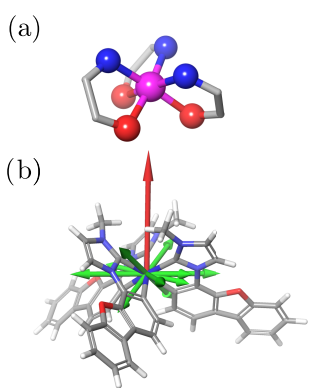

facial $(f a c)$

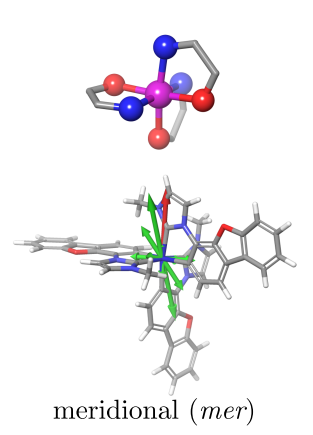

meridional (mer)
Figure 1. (a) Schematic representation of the facial (fac) and meridional (mer) isomers of an $\operatorname{Ir}\left(\mathrm{C}^{\wedge} \mathrm{N}\right)_{3}$ complex. While the facial complex has a $\mathrm{C}_{3}$-symmetry axis, the meridional isomer shows no symmetry. Note that both the meridional and facial complexes are chiral, which is not further considered here. (b) Optimized geometry of the facial and meridional reference complexes $\operatorname{Ir}(\mathrm{dbfmi})_{3}$ and their PDM (red) and TDMs (green), calculated with TD-DFT. Details on the computational method are available in Section 6. While the PDM and the TDMs are nearly perpendicular to each other for the facial complex, they lie almost in the same plane for the meridional complex.

rotational $\left(\mathrm{C}_{3}\right)$ symmetry, its meridional counterpart has just the trivial $\left(\mathrm{C}_{1}\right)$ symmetry. Remarkably, most studied Ir -complexes for the application in OLEDs have the facial configuration. ${ }^{10}$ Due to their symmetry, these compounds are likely to exhibit three equivalent transition dipole moments, one for each ligand. ${ }^{23}$ On the one hand, this makes it experimentally more challenging to address the actual molecular orientation, as all transitions contribute to the optically accessible mean orientation value. ${ }^{21,23}$ On the other hand, for certain configurations of the TDMs, the film may appear isotropic although the molecules are all aligned, e.g., if the three TDMs are mutually orthogonal. ${ }^{14,18}$ For meridional complexes, the situation is different. As the molecules only have $\mathrm{C}_{1}$ symmetry, a single ligand can be responsible for light emission, leading to a dominant TDM for each molecule. In general, the two isomers of the same compound can exhibit different physical properties, like emission spectrum, quantum efficiency, and stability. ${ }^{24,25}$ In this study, we combine optical and electrical measurements of the N-heterocyclic carbene complex tris $(N$-dibenzofuranyl- $N$ '-methylimidazole)iridium(III) $\left(\operatorname{Ir}(\mathrm{dbfmi})_{3}\right)$ and derivatives of this sky-blue dye in two wide-gap hosts, namely, bis[2-(diphenylphosphino)phenyl]ether oxide (DPEPO) and 3,6-bis(diphenylphosphoryl)-9phenylcarbazole (PO9). Interestingly, this homoleptic phosphorescent emitter shows a significant alignment of both its TDMs and its PDM. From the detailed insights into the film morphology, we propose that two factors control the alignment of the dye: on the one hand, the aspect ratio of the dye provides a geometrical constraint for the preferred orientation on the film surface. On the other hand, local electrostatic interactions of the evaporated emitter molecules with the host may play an important role in the orientation process as well.

\section{QUANTUM CHEMICAL CALCULATIONS}

Figure 2 shows the chemical structure of $\operatorname{Ir}(\mathrm{dbfmi})_{3}$ and all derivatives investigated in this study. Their synthesis and photophysical characterization are described elsewhere. ${ }^{26}$ To get insight into the molecular geometry and properties, we performed quantum chemical calculations for all investigated complexes. Details on the computational parameters are described in Section 6. The electrostatic surface potentials (ESPs) of all meridional dyes and their permanent dipole moments are also shown in Figure 2a. In addition to the displayed meridional structures, we studied the facial configuration for the reference complex $\operatorname{Ir}(\mathrm{dbfmi})_{3}$ and the modifications A-1 and A-5. Their ESPs and PDMs are shown in Figure 2b. From the shown heat maps, it becomes clear that the different modifications have an impact on the electrostatic surface. Especially, the ESP maximum, which is located near the two nitrogen atoms of the imidazole group (blue region), changes with the substitutions. For a more quantitative analysis of the ESP, the maxima, minima, and mean values are shown in Table S1.

By contrast, the PDMs of the different meridional complexes are only slightly affected by the substitutions, except for the structure B-2, which exhibits a PDM of $11.4 \mathrm{D}$-more than twice the value of the reference dye $\operatorname{Ir}(\mathrm{dbfmi})_{3}$. A change of the symmetry of the compounds has a larger impact on the polarity, as the facial isomers always show a larger PDM compared to their meridional counterparts. More precisely, the calculation yields values of $7.8 \mathrm{D}$ for $f a c-\operatorname{Ir}(\mathrm{dbfmi})_{3}, 8.1 \mathrm{D}$ for fac-A-2, and $8.7 \mathrm{D}$ for fac-A-5, which is an increase of more than $50 \%$.

Furthermore, we performed time-dependent density functional theory (TD-DFT) calculations for the complexes to evaluate the direction of the TDMs with respect to the molecular frame. An exemplary result for the facial and meridional $\operatorname{Ir}(\mathrm{dbfmi})_{3}$ is shown in Figure 1; for a complete overview of all structures, refer to Figure S2. We cannot identify a single TDM that is responsible for light emission, as the calculation yields many transitions with similar energy and oscillator strength. Interestingly, all TDMs of a given complex are nearly in-plane and are not largely altered by the substitution at the ligand. For the facial configuration, the transitions are almost perpendicular to the PDM and the rotational symmetry axis, while PDM and the TDMs are nearly coplanar in the case of the meridional counterpart.

To further analyze the molecular geometry, we calculated the smallest bounding box for all of the geometries with the Schrodinger Materials Science Suite. ${ }^{27}$ The aspect ratio $\gamma$ is defined according to

$$
\gamma=\frac{\sqrt{a \cdot b}}{c}
$$

where $a$ and $b$ describe the length and the width of the base, respectively, while $c$ corresponds to the smallest edge of the unit cell. The meaning of the different parameters is additionally clarified in Figure 3 a. From the resulting values shown in Figure $3 \mathrm{~b}$, it becomes clear that the aspect ratio of the complexes deviates from 1 , which would refer to a perfectly isotropic shape, like a sphere or a cube. This indicates a disklike shape of the dye molecules. Nevertheless, the aspect ratio is relatively small (between 1.2 and 1.9) as compared to other organic compounds that show a high degree of orientation. ${ }^{8}$

\section{EXPERIMENTAL RESULTS}

In our experimental study, we fabricated guest-host systems of all complexes in the hosts DPEPO and PO9 by thermal coevaporation at a volume concentration of about $8 \%$. Details on the film fabrication are described in Section 6. We first investigated the photoluminescence spectra (see Figure S1) and the excitonic lifetimes (see Figure 4a) of the compounds. From these results, it becomes clear that the photoluminescence does not dependent on the chosen 
(a)

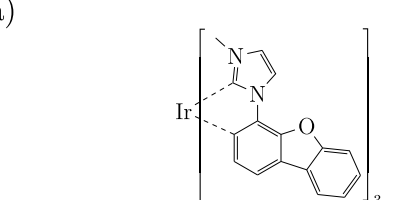

$\operatorname{Ir}($ dbfmi $)$

PDM: 4.9 D
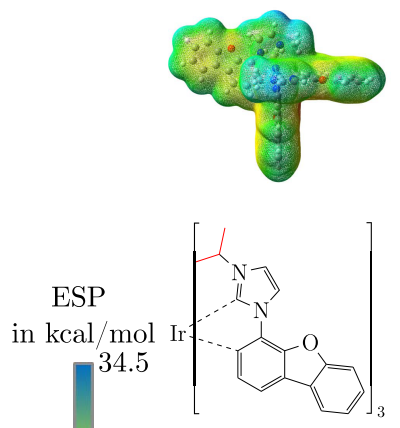

A-3

PDM: $5.0 \mathrm{D}$
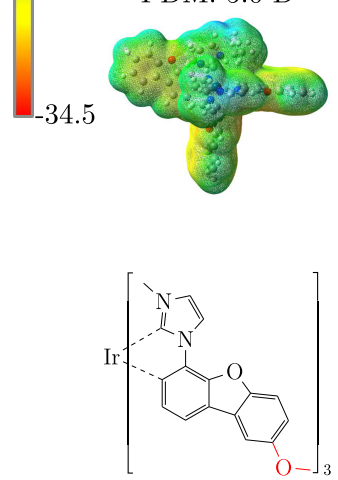

B-1

PDM: 5.5 D

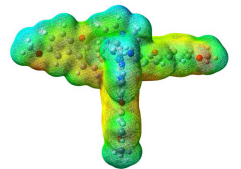

ESP

(b)

in kcal / mol

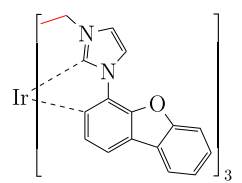

A-1

PDM: $5.0 \mathrm{D}$
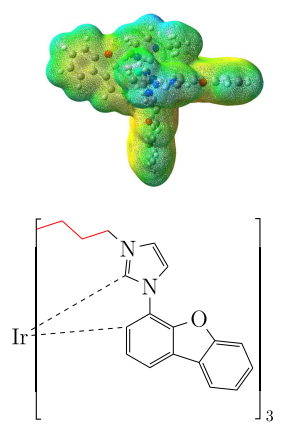

A-4

PDM: $5.0 \mathrm{D}$
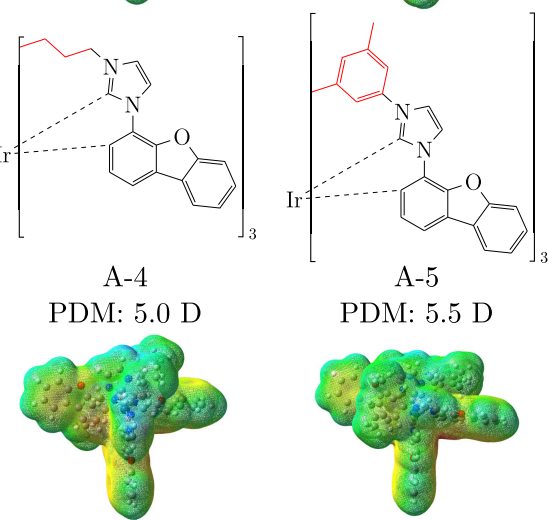

A- 5

PDM: $5.5 \mathrm{D}$
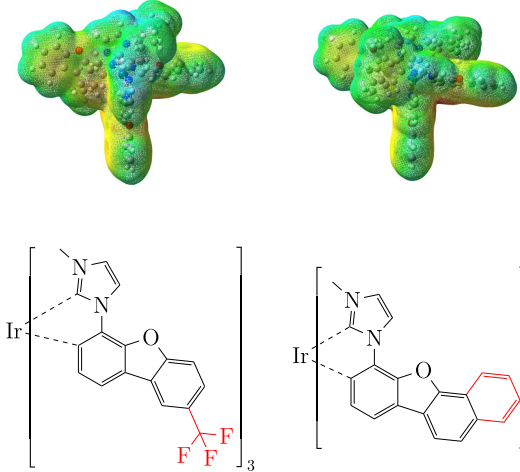

B-2

PDM: 11.4 D
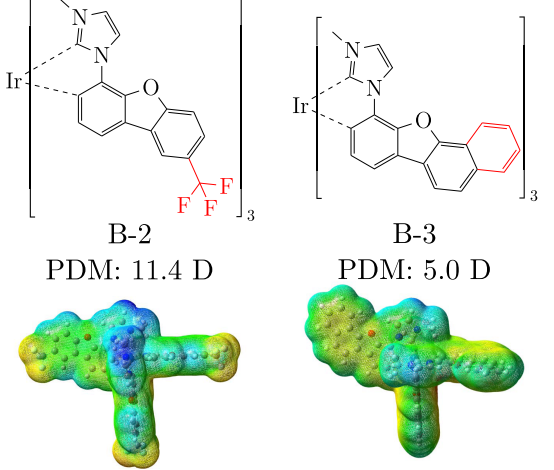

B-3

PDM: $5.0 \mathrm{D}$
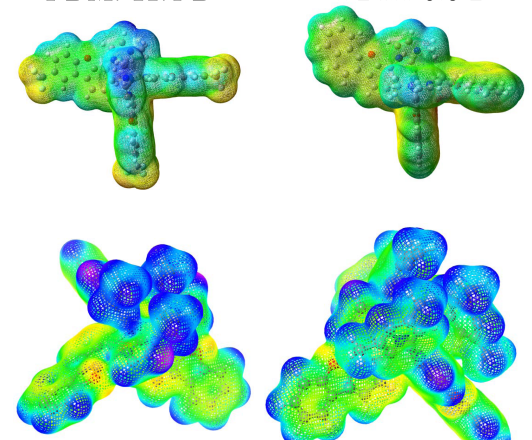

fac-A-1

PDM: $8.0 \mathrm{D}$

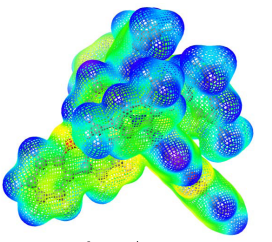

fac-A-5

PDM: 8.7 D

Figure 2. Chemical structures and ESP maps of the (a) meridional and (b) facial Ir complexes investigated in this study. For the compounds A-1A-5, the imidazole group was modified with different alkyl chains. The dibenzofuran was modified for the materials B-1-B-3. The ESP maps were calculated with DFT (details in Section 6). An isovalue of 0.004 a.u. for the electron density was chosen for the mapping. The facial configuration was additionally studied for the reference dye $\operatorname{Ir}(\mathrm{dbfmi})_{3}$ as well as the compounds A-1 and A-5.

host. Neither the spectra nor the lifetime differ for a dye, when doped in DPEPO or PO9. Additionally, the spectra and lifetime of all compounds, except for the complex B-3, are similar. These results are in line with our TD-DFT calculations. As the TDMs are similar with respect to the direction and strength of all molecules except for B-3, a similar phosphorescence is expected. The TD-DFT calculations also allow for conclusions about the phosphorescence lifetime, ${ }^{28}$ which is plotted together with the experimental values in Figure 4. The resulting values agree almost quantitatively with the measurement, even for the high phosphorescence lifetime of the compound B-3. The similar behavior of the dyes (except for B-3) can be explained by the fact that the substitutions at the ligands do not directly alter the aromatic system of the ligand compared to the reference dye. For this reason, the chromophoric system is not largely altered in these cases, and the TDMs of these dyes are comparable with respect to strength and the direction on the molecular frame if dyes of the same symmetry are considered. So the comparison of the average orientation of the TDM allows for conclusions about morphology in the film. By contrast, modification B-3 is characterized by benzannulation of the dibenzofuran, which results in an additional 


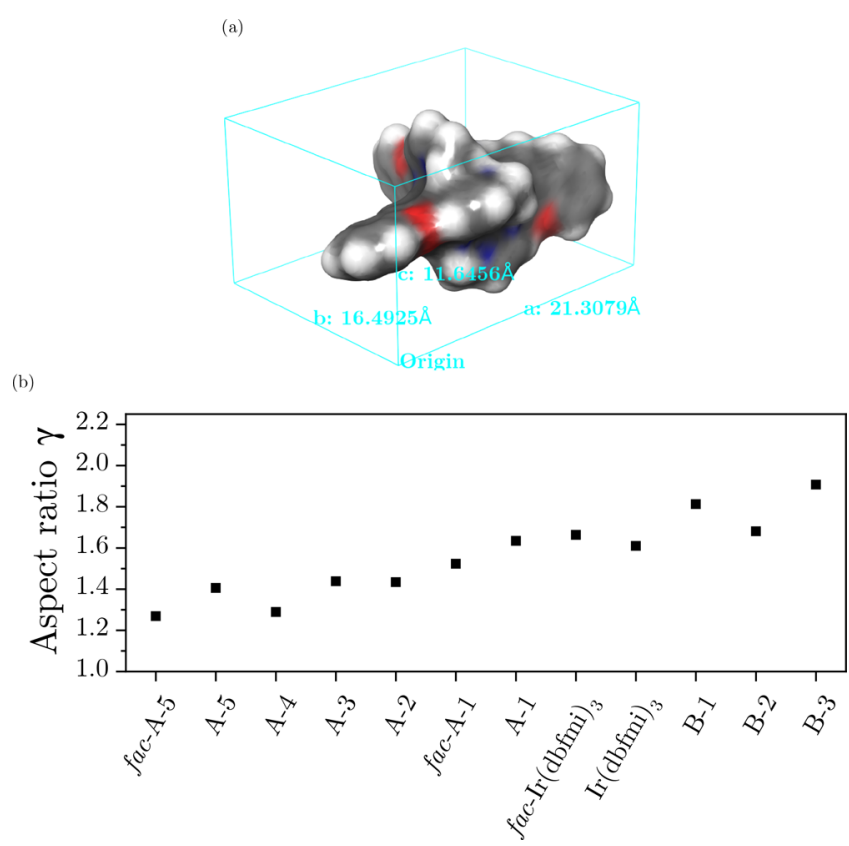

Figure 3. (a) Exemplary bounding box of $m e r-\operatorname{Ir}(\mathrm{dbfmi})_{3}$. A cuboid with the minimum possible volume including the full molecule has been chosen to allow for the calculation of the aspect ratios. The colors of the surface here represent the element type: carbon: gray, nitrogen: blue, oxygen: red, and hydrogen: white. (b) Resulting aspect ratios for all studied derivatives. All of them are meridional unless otherwise noted.

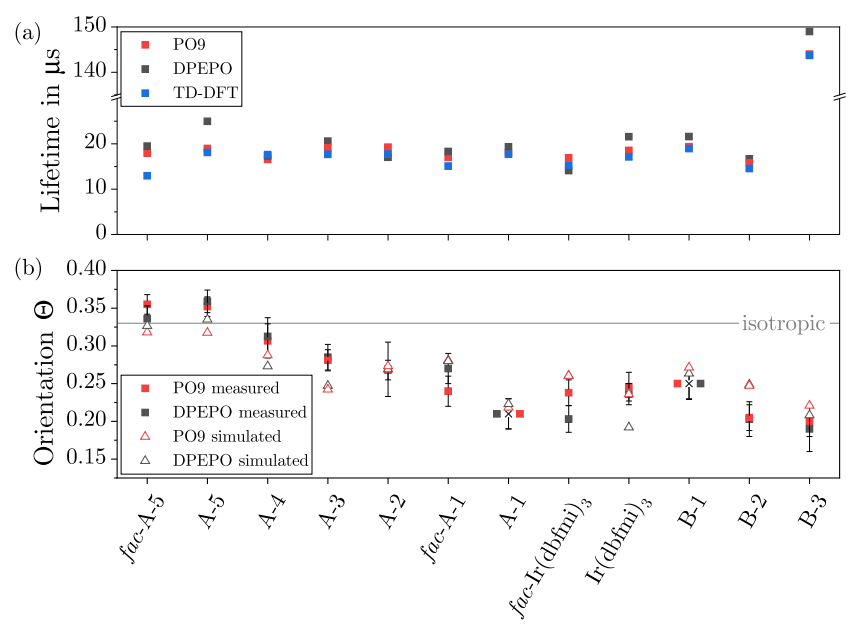

Figure 4. (a) Measured and calculated phosphoresce lifetimes and (b) orientation values of the investigated complexes in DPEPO and PO9. While the orientation varies for the different derivatives, the lifetime is hardly influenced except for dye B-3, where major changes of the aromatic system were performed. The raw data from ADPL and transient PL together with the fits leading to the shown values are available in the Supporting Information.

ring connected to the chromophoric system. The larger aromatic system leads to a pronounced bathochromic shift of the emission. Nevertheless, the direction of the TDMs is similar to one of the other meridional dyes.

We measured the average orientation of the transition dipole moment with ADPL, as described in the literature. ${ }^{6}$ We chose the fraction of vertical dipoles

$$
\Theta=\frac{\sum p_{\mathrm{z}}^{2}}{\sum p_{\mathrm{tot}}^{2}}
$$

as the order parameter for the orientation, where $\sum p_{\mathrm{z}}^{2}$ represents the sum of overall TDM components perpendicular to the substrate and $\sum p_{\text {tot }}^{2}$ represents the total sum of all TDMs. Figure $4 \mathrm{~b}$ shows the results for all different dyes in the chosen hosts; raw data from ADPL and the respective fits for the determination of $\Theta$ are shown in Figure S3. Details of the measurement procedure are described in Section 6. Again, no dependence on the host is observable. Importantly, the substitution at the ligand has a strong impact on the average TDM alignment. Especially, the modifications at the imidazole group (compounds A1-A5) lead to systematic changes. The larger the attached side group is, the less horizontal orientation is observed, leading to an isotropic or even slightly vertical TDM for the compounds fac-A-5, A-5, and A-4. The substitutions at the dibenzofuran unit $(\mathrm{B} 1-\mathrm{B} 3)$ have a smaller impact on the film morphology. The order parameter $\Theta$ decreases for these dyes but conclusions about the mechanism are not obvious from the optical measurement alone.

Additionally, we performed atomistic simulations to reproduce the orientation process. In this calculation, the deposition is modeled by a Monte Carlo-based simulated annealing protocol. ${ }^{29}$ Further details are described in Section 6. This model has successfully reproduced the orientation of other organic phosphors previously. ${ }^{18}$ As shown in Figure $4 \mathrm{~b}$, the simulated TDM orientation $\Theta$ agrees with the experimental values in our study as well. The observed trends are reproduced in general, and no major difference in the alignment is observed for the two different hosts.

For further insights into the film morphology, we additionally carried out impedance spectroscopy measurements. Materials with a PDM that show preferential alignment exhibit a macroscopic spontaneous orientation polarization (SOP), often also termed giant surface potential (GSP). This phenomenon has originally been found in neat films of polar molecules but can even persist in diluted guesthost systems. ${ }^{21,30}$ In capacitance-voltage characteristics, the concomitant interface charge toward a nonpolar layer can be evaluated quantitatively. ${ }^{30-32}$ This allows for conclusions about the average distribution of PDMs in the film. ${ }^{30,33,34}$ Due to the high demand for material, these measurements were only carried out on the compounds $\operatorname{Ir}(\mathrm{dbfmi})_{3}, f a c-\operatorname{Ir}(\mathrm{dbfmi})_{3}, f a c-A-5$, and B-2, as these complexes show the greatest differences in the optical experiments and a large PDM. The neat host DPEPO and a mixture of DPEPO with $N, N^{\prime}$-Bis(naphthalen-1-yl)- $N, N^{\prime}$-bis(phenyl)benzidine (NPB) were additionally investigated for comparison. All doped films were fabricated with a volume concentration of about $8 \%$ in consistency with the optical experiments. Further details on the sample preparation and the measurement setup are described in Section 6 . Figure 5a shows the measured interface charge densities for the different material systems. The neat matrix DPEPO shows SOP with an interfacial charge density of $1.68 \pm 0.05 \mathrm{mC} \mathrm{m}^{-2}$. This behavior has been observed for many polar materials. ${ }^{34}$ A small fraction of PDMs orients with its negative partial charge pointing toward the substrate and is not compensated by other PDMs in the film. This leads to a macroscopic polarization of the film, as shown in Figure $5 \mathrm{~b}$ (top). Upon doping the dyes $\operatorname{Ir}(\mathrm{dbfmi})_{3}, f a c$ - $\operatorname{Ir}(\mathrm{dbfmi})_{3}$, and B-2 into the matrix, the interfacial charge density is reduced to $1.55 \pm 0.05$, $1.08 \pm 0.08$, and $1.17 \pm 0.07 \mathrm{mC} \mathrm{m}^{-2}$, respectively. We attribute this effect to a mutual compensation of the PDMs of DPEPO and the Ir complexes, as schematically shown in Figure $5 b$ (bottom left). More precisely, the matrix aligns with its PDM pointing toward the film surface, causing a negative charge density at interface with the hole transport layer, while the PDMs of the dye molecules are pointing in the opposite direction. Thereby, they partially compensate the host's GSP. Interestingly, the alignment of the PDM causing a negative charge density at the underlying film is frequently observed, while the opposite behavior is extremely rare. Only a few materials are known to cause a polarization with a positive sign., ${ }^{1,34,35}$ We additionally confirmed these findings with Kelvin probe for the neat $\operatorname{Ir}(\mathrm{dbfmi})_{3}$ 
(a)

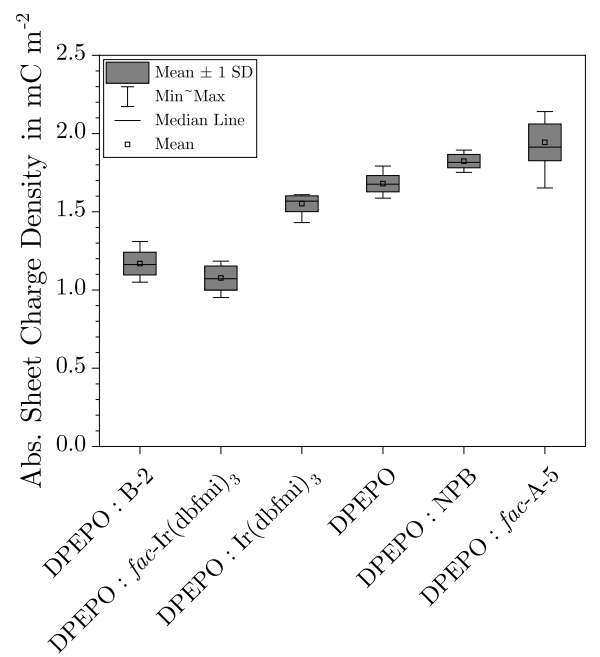

(b)

(1) Neat host
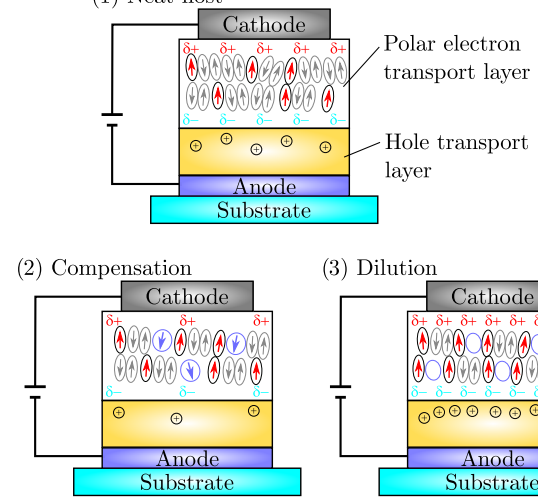

(3) Dilution

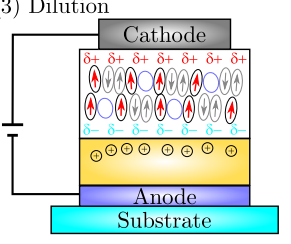

Figure 5. (a) Absolute value of the interfacial charge density of neat DPEPO and DPEPO doped with the five guests $\operatorname{Ir}(\mathrm{dbfmi})_{3}, f_{a c-I r}(\mathrm{dbfmi})_{3}, f_{a c}$ A-5, B-2, and NPB. The error results from the statistics of all working pixels (maximum 4) on at least four different samples. (b) Sketch of the device structure and the morphology in the polar layer. (1) Neat DPEPO: a small fraction of uncompensated PDMs (red) leads to a negative interfacial charge to the adjacent HTL. (2) DPEPO doped with $\operatorname{Ir}(\mathrm{dbfmi})_{3}, f a c-\operatorname{Ir}(\mathrm{dbfmi})_{3}$, or B-2: the dye molecules align with their PDM (blue) pointing in the opposite direction than the PDMs of the host molecules, leading to a smaller polarization. (3) DPEPO doped with NPB or fac-A-5: due to the dilution of the host molecules, there is a higher fraction of uncompensated PDMs of the host, resulting in a higher overall charge density. Interestingly, this effect seems to occur for both the polar fac-A-5 as well as the nonpolar NPB.
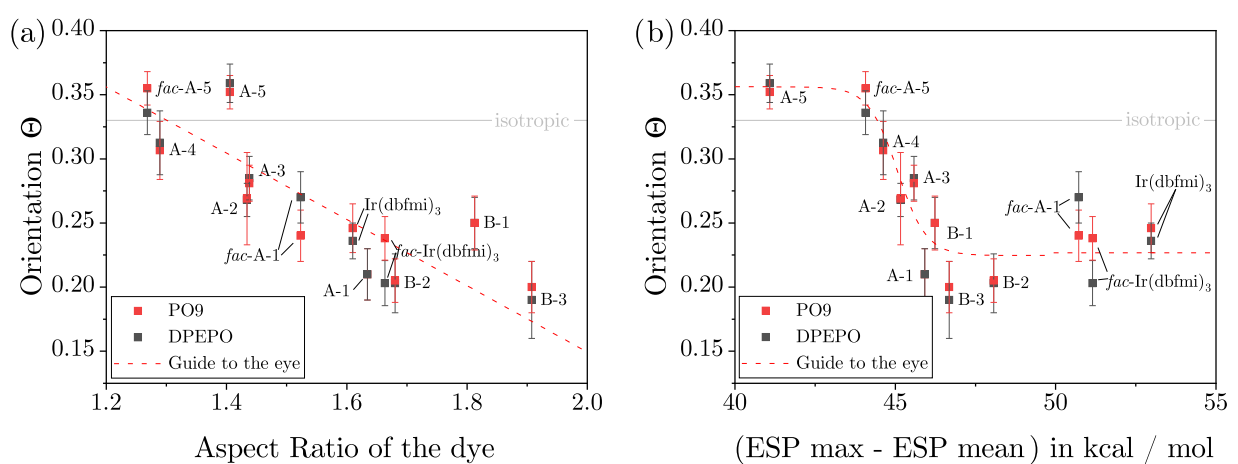

Figure 6. Measured TDM orientation $\Theta$ in dependence of the (a) aspect ratios and the (b) difference between the maxima and mean values of the ESP of the dyes.

dye, as a positive charge density at the interface with the hole transport layer (HTL) is not directly observable with impedance spectroscopy. The measurement results and a brief description of this technique are available in Section 6 and shown in Figure S7, respectively. The investigated film shows a negative GSP slope, indicating a significant orientation of the PDMs in the same direction as observed with impedance spectroscopy in the doped film.

The GSP compensation on the host varies strongly for the different complexes. While the change is rather small for the meridional reference dye $\operatorname{Ir}(\mathrm{dbfmi})_{3}$, the facial counterpart causes the largest change of the GSP. There are two reasons for this effect. First of all, the PDM of the facial complex $(7.8 \mathrm{D})$ is larger than the one of the meridional isomer $(4.9 \mathrm{D})$. Additionally, for the facial complex, the TDM and the PDM are nearly perpendicular to each other. From the horizontal orientation of the TDM observed for this dye, it thus becomes clear that the PDM has to be mostly perpendicular to the substrate. So the PDM contributes with a large out-of-plane component to the compensation of the host's GSP. By contrast, the PDM and the TDMs of the meridional isomer lie nearly in the same plane. From the optical measurement, it is clear that this plane is predominantly aligned parallel to the substrate. Thus, every PDM can only contribute with a small fraction to the overall compensation. The finding for the meridional compound B-2 is consistent with this model, as it exhibits the largest PDM of 11.4 D. Therefore, this dye is able to reduce the interfacial charge density of DPEPO more strongly than the meridional complex $\operatorname{Ir}(\mathrm{dbfmi})_{3}$. Due to the direction of the PDM being nearly coplanar with TDMs, the overall interfacial charge density is in the same range as for $f a c-I r(d b f m i){ }_{3}$ in spite of their difference of the PDMs.

Upon doping the DPEPO matrix with the nonpolar NPB and the dye fac-A-5, we observe a different behavior. Instead of a compensation and a reduction of the sheet charge density, the magnitude increases slightly to higher absolute values of $1.82 \pm 0.05$ and $1.94 \pm 0.12 \mathrm{mC} \mathrm{m}^{-2}$, respectively. This behavior has already been observed for other polar materials as well if they are diluted with a nonpolar, organic species. ${ }^{30,36}$ Due to dilution, the dipole-dipole interactions between the polar host molecules are reduced, decreasing the driving force for an antiparallel alignment of their PDMs. This leads to a higher net polarization, in spite of a lower content of polar host molecules. ${ }^{30,36}$ This behavior is schematically shown in Figure $5 \mathrm{~b}$ (bottom right). Remarkably, the increase of the charge density is similar for doping of DPEPO with the nonpolar NPB and the fac-A-5 dye, which exhibits a large dipole moment of $8.7 \mathrm{D}$. Therefore, we attribute the increase of the GSP to the above-described dilution effect. In combination with the results from angular-dependent photoluminescence, where an almost isotropic alignment is observed for this dye, we conclude that the Ir complex fac-A-5 is randomly oriented within the host matrix. This leads to the question why the 
(a)

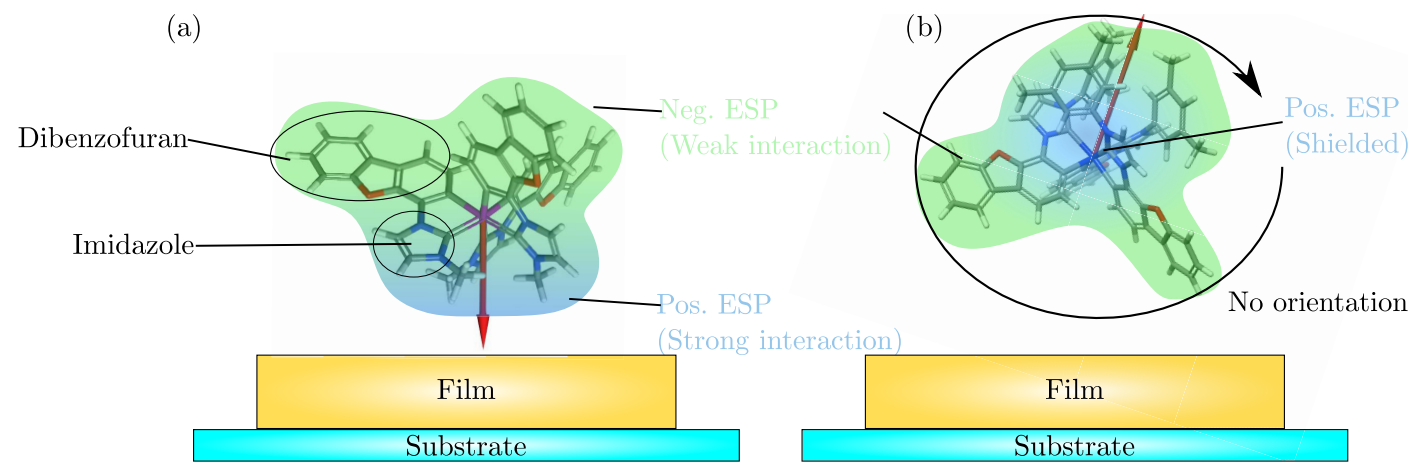

Figure 7. Schematic representation of the interaction of (a) fac- $\operatorname{Ir}(\mathrm{dbfmi})_{3}$ and (b) fac-A-5 during the deposition process. In the case of fac$\operatorname{Ir}(\mathrm{dbfmi})_{3}$, the imidazole group is able to strongly interact with the previously deposited molecules, leading to a preferential alignment of the dye in the film. For fac-A-5, the imidazole group is shielded from its surrounding by the additional phenyl rings. This leads to an isotropic distribution of the molecules within the film.

different compounds and, particularly $f a c-\operatorname{Ir}(\mathrm{dbfmi})_{3}$ and fac-A-5, exhibit such a different orientation behavior and if we can relate their different behaviors to their molecular structures.

\section{DISCUSSION}

In previous work, the orientation of Ir complexes was brought in context to the PDM, ${ }^{22}$ and molecular-scale simulation of the film formation suggested that dipole-dipole interactions tend to hinder alignment in organic films. ${ }^{18,19}$ In this study, no such correlation can be identified, as shown in Figure S4, where $\Theta$ is plotted in dependence of the PDM for all investigated complexes. While most meridional isomers with a modification at the imidazole group show a similar PDM, they exhibit quite different orientations of their TDMs. In addition, the compound B-2, showing the strongest orientation of its TDMs, is nearly twice as polar as the other meridional components. If the polarity of the dye indeed counteracted the alignment, as stated previously, we would expect the opposite behavior. Therefore, we conclude that dipole-dipole interactions do not play a significant role in the orientation of these complexes.

For organic molecules, their shapes have been identified as a key parameter for alignment in vapor-deposited thin films. ${ }^{8,10,14}$ Indeed, the complexes in this study show an aspect ratio greater than 1 , indicating a disk-like shape. Figure $6 \mathrm{a}$ shows the orientation of the complexes in dependence of the aspect ratio. From this graph, it becomes clear that the emitters with a larger aspect ratio are generally stronger aligned, resulting in smaller values of $\Theta$. But the two dyes A-5 and B-1, deviate from this general trend as their $\Theta$ values are higher than expected. Therefore, we also analyzed the ESP of the complexes. As shown in Figure 6b, the TDM orientation is plotted against the difference between the maximum and the mean value of the ESP on an isosurface (isovalue $=0.004$ a.u.) of the electron density. Although all information about the spatial distribution of the potential is lost by only considering the mean and the maximum values, it becomes clear that the TDM orientation also correlates with the ESP. For the dyes with a small difference between maximum and minimum, no orientation is observed. The $\Theta$ value decreases rapidly with increasing difference and seems to saturate at a value of about 0.22 .

Apparently, the correlation between TDM orientation and the aspect ratio (Figure 6a) is much simpler than between $\Theta$ and ESP (Figure 6b). Thus, one can identify the emitter's molecular aspect ratio as the main driver for interface alignment during film growth. However, based on this simple geometric model alone, there is no obvious reason for the observed asymmetry in the alignment of the PDMs of the facial complexes, i.e., their spontaneous orientation polarization or GSP. This indicates that the local ESP plays a role in alignment as well, as will be discussed in the following.

From the compensation of the negative sheet charge density of the host material observed in the capacitance-voltage measurement, it becomes clear that the PDM of $f a c-\operatorname{Ir}(\mathrm{dbfmi})_{3}$ is pointing toward the substrate in the film. This, in turn, means that the imidazole group of the molecule also aligns toward the substrate during film growth, as schematically shown in Figure 7a. Thus, the interaction of this group with the underlying film has to be stronger than the interaction of the rest of the molecule, namely, the dibenzofuran group. Therefore, we propose that this region of the molecule shows a strong local interaction with the host molecules due to its electron deficiency and its maximum of the ESP. Taking this additional effect into account, we can explain the formation of the macroscopic polarization and the outliers (compounds A-5 and B-1) from the general trend of the aspect ratio.

Upon substitution of the methyl group at the imidazole with xylene (compound A-5), the lack of electrons at this group is reduced by this comparably electron-rich substituent. Additionally, the imidazole group, where the electron-deficient region was located before, is no longer exposed to the surface of the molecule, leading to a comparably uniform ESP map (with a small difference between the maximum and the mean value), as shown in Figures 2 and $6 \mathrm{~b}$. Thus, the center for the strong interaction is now shielded from interacting with the previously deposited host molecules, as schematically shown in Figure $7 \mathrm{~b}$. For this reason, no molecular orientation is observable for the Ir complex A-5 (both facial and meridional). The optical results for the other complexes A-1-A-4 are consistent with the proposed mechanism. The length of the attached alkyl group has two effects: on the one hand, the aspect ratio decreases, and on the other hand, the ESP maximum shifts closer to the mean value. Therefore, less orientation is observed in the series A-1-A-4.

For modification B-1 at the dibenzofuran group, the effect is more subtle as the substitution is spatially more separated from the direct center of the interaction. From the aspect ratio alone, we would expect a more horizontal alignment, while the difference between ESP maximum and ESP mean seems not to account for the high $\Theta$ value. In this case, the electrondonating methoxy group was attached to the dibenzofuran. As 
shown in Figure 2, this has two effects on the ESP surface of the complex. First of all, it slightly reduces the electron deficiency at the imidazole, probably reducing its interaction with the surrounding molecules during film formation. More importantly, the methoxy group itself introduces a second electron-deficient center for interaction between the dye and host molecules that competes with the imidazole, as it is located on the opposite side. Thus, the effect of this substitution is not fully covered by the evaluation of the maximum and the mean ESP, causing the comparably isotropic orientation of this dye. The other two complexes with a modification at the dibenzofuran group exhibit the smallest $\Theta$ values. They seem to profit from their high aspect ratio in comparison with the other emitters.

Finally, we want to put our results in the context of previously published Ir complexes. For heteroleptic molecules, different mechanisms were suggested to explain their observed alignment. Jurow et al. ${ }^{23}$ proposed that the orientation is based on an anisotropic interaction of the molecules caused by different polarizabilities of the ligands. While aromatic regions of the molecule interact strongly with the underlying film during growth, aliphatic molecular ligands orient toward the vacuum. This leads to an overall anisotropy of the film during vacuum deposition. For the dyes studied here, this mechanism seems to play a minor role, as most complexes are fully aromatic. For the compounds having an aliphatic part (A-1-A4 ), the mechanism proposed by Jurow et al. would lead to an alignment of the imidazole group toward the vacuum. By contrast, the local electrostatic interactions with the film surface tend to align this group toward the substrate. By increasing the size of the aliphatic region of the molecule, the electrostatic interaction is reduced, while the difference in polarizability is increased. Thus, the mechanism of Jurow et al. might contribute to the observed trend that the alignment of the dye is reduced by increasing the length of the attached alkyl chain at the imidazole as the interaction due to the different polarizabilities compensates the electrostatic interactions.

In the literature, the role of dipole-dipole interactions for the orientation is also discussed frequently. ${ }^{18,19,22}$ Here, we do not observe a pronounced dependence of the molecular orientation on the PDM of the structures. Therefore, we conclude that dipole-dipole interactions only play a subordinate role in the orientation. According to Kim et al., ${ }^{14,37}$ the orientation is mostly related to attractive electrostatic interactions between the heteroleptic complexes and the host molecules. From the study, here, we draw similar conclusions and suggest that this mechanism is present in both heteroleptic and homoleptic complexes. In addition, we do not observe a dependence of the average TDM alignment on the host, while other studies reveal a slight dependence on the host molecule. ${ }^{38,39}$ Please note that a dependency cannot be fully ruled out in our study, as the two hosts are quite similar. However, only a few materials are suitable for the emitters studied here as they require high triplet energy of the host for efficient luminescence. Apart from the complexes in this study, only few homoleptic Ir complexes are known to show a pronounced orientation of their TDM, namely, tris (1phenylisoquinoline)iridium(III)( $\left.\operatorname{Ir}(\text { piq })_{3}\right)$, tris (2-(1-cyclohexenyl)pyridine)iridium(III) $\left(\operatorname{Ir}(\text { chpy })_{3}\right)$, and tris3-(4-(tertbutyl)pyridin-2-yl)-2,6-diuorobenzonitrile-iridium(III)( $\mathrm{Ir}(\mathrm{tBu}-$ FCN $\left.)_{3}\right)^{10,14}$ Different mechanisms were suggested to be responsible for the alignment of these molecules. However, no systematic modifications to their structures were made to identify the more or less dominant effects.

\section{CONCLUSIONS}

In conclusion, we studied the sky-blue homoleptic Ir complex $\operatorname{Ir}(\mathrm{dbfmi})_{3}$ in both facial and meridional configurations as well as its multiple derivatives. By attaching different functional groups to the reference dye, we were able to show that the orientation is strongly influenced by different side groups. We conclude that two effects are relevant for the alignment: the aspect ratio, which has been identified as a key parameter for fluorescent molecules ${ }^{8,10,40}$ plays an important role in the overall alignment. Additionally, local electrostatic interactions of the dye molecule with the film surface during deposition are responsible for the formation of interfacial polarization and have an impact on the overall orientation as well, as has been observed in heteroleptic Ir complexes. ${ }^{14,37}$ The combination of both mechanisms is able to fully describe the qualitative orientation behavior. We anticipate that developing more detailed guidelines for optimizing their structures further is necessary for achieving a higher degree of orientation for the next generation of Ir complexes.

\section{METHODS}

6.1. Material Abbreviations. The following materials were bought in a sublimed grade of purity from the specified supplier: NPB, $N, N^{\prime}$-bis(naphthalen-1-yl)-N,N'-bis(phenyl)benzidine (Lumtec); DPEPO, bis[2-(diphenylphosphino)phenyl]ether oxide (Lumtec or TCI; no detectable difference between the material of the different suppliers); ITO, indium tin oxide (purchased from Thin Film Devices); and PEPDOT:PSS, poly(3,4-ethylenedioxythiophene):polystyrene sulfonate (CLEVIOS P VP AI 4083).

The other materials were synthesized at the Technical University of Braunschweig: PO9, 3,6-bis(diphenylphosphoryl)-9-phenylcarbazole (synthesis according to literature $\left.{ }^{41}\right)$; and $\operatorname{Ir}(\mathrm{dbfmi})_{3}$ and derivatives, tris( $N$-dibenzofuranyl- $N$ '-methylimidazole)iridium(III) (details on synthesis in ref 26).

6.2. Sample Preparation. All samples were fabricated under high vacuum conditions at pressures between 2.0 and $15 \times 10^{-7} \mathrm{mbar}$ by thermal evaporation. For optical measurements, the film was deposited on precleaned BK7 glass substrates. The impedance and Kelvin probe samples were fabricated on glass substrates with a 150 nm ITO layer. Thirty nanometers of PEDOT:PSS was optionally coated on the substrates under clean-room conditions for hole injection before the evaporation of organic layers and contacts.

6.3. DFT Calculations. We performed a geometry optimization of the Ir complexes in their electronic ground state in vacuum at the B3LYP and LANL2DZ ${ }^{42}$ level of theory with Gaussian $09 .{ }^{43}$ The given PDMs and ESPs were obtained for these optimized structures. Thereafter, we reoptimized the structures with Turbomole Version 7.4.1 $1^{44,45}$ with the same basis set and functional with def-ecp effective core potentials (ECPs) for the Ir atom from ref 46. These results were consistent with the calculations from Gaussian, except for complex B-1, where the different programs converge to slightly different geometries. Probably, this dye exhibits two conformations with slightly different PDMs. To obtain the TDMs, we performed TD-DFT with Dalton $2018^{47,48}$ on the ground-state geometries obtained by the optimization with Turbomole. We used the B3LYP functional and exp-sdd-DZ basis set in combination with the previously mentioned ECPs for iridium and the $3-21 \mathrm{G}$ basis set for all other elements. The effects of spin-orbit coupling are included in the phosphorescence calculation of the first 10 excited states with the effective charge approximation for the spin-orbit operator. ${ }^{4-51}$ This yielded three separate TDMs for each excited state. From their magnitude and their relative energy, we calculated the predicted phosphorescence lifetime of the complexes according to the method published by Mori et al. ${ }^{28}$ 
6.4. Deposition Simulations. 6.4.1. Structure Generation with DEPOSIT. We generated mixed films of all measured host and emitter combinations with the Metropolis Monte Carlo approach published by Neumann et al. ${ }^{29}$ It has been shown that this method is able to predict effects like the emitter orientation of phosphors ${ }^{18}$ as well as the GSP of neat films. ${ }^{33}$ The following simulation parameters were kept fixed for every simulation:

- $T_{\text {high }}=4000 \mathrm{~K}$

- $T_{\text {low }}=300 \mathrm{~K}$

- $T_{\text {acc }}=300 \mathrm{~K}$

- number of Monte Carlo steps: 300000

- number of self-annealing cycles: 30

- boxsize in the $X / Y$ direction: $10 \mathrm{~nm}$

- number of deposited molecules: 1200

To parametrize the torsions, each dihedral angle was rotated around $360^{\circ}$ in steps of $20^{\circ}$. At each step, the energy was evaluated with density functional theory (DFT) using Turbomole Version 7.4.1 $1^{44,45}$ at B BLYP and def2-SVP level of theory. In combination with the DFT results, we fitted a set of internal forcefield parameters for the nonbonded interactions against 250 random training configurations.

To estimate the molar volume of each molecule, we first generated pure films of each material and calculated the density of each film. This information was then used to calculate the molecular deposition ratios for each host-emitter combination corresponding to a volume concentration of $8 \%$.

6.4.2. Analyzing the Emitter Orientation. For each guest-host system, five independent films were generated for the analysis of the emitter orientation. We mapped the TDM calculated by TD-DFT (see Section 6.3) for the simulated morphologies. This was done by first calculating the rotation matrix, which rotates the coordinate system defined by the central Ir atom and the three innermost $\mathrm{C}$ atoms of the conformer used in the TD-DFT calculation for the specific conformer of the simulated film. With this mapping approach, we neglect potential changes to the TDM through dihedral rotations of the outer ligand groups.

The final orientation parameter of a film was then calculated by averaging the $\cos \theta_{\mathrm{Z}}$ value for each TDM over all emitters in the film. ${ }^{23}$ In our case, this yielded 30 different orientation parameters from which we computed the Boltzmann weighted average according to their transition energies at $T=300 \mathrm{~K}$. Finally, we averaged this value again over all five independent films to minimize statistical fluctuations.

6.5. Angular-Dependent Photoluminescence. Angular-dependent photoluminescence measurements were carried out as described in previous work by us. ${ }^{6}$ In the setup, the sample is excited with a focused laser (Kimmon $\mathrm{HeCd}$ ) at a wavelength of $325 \mathrm{~nm}$ normal to the substrate plane. The samples are fixed to a fused-silica prism with index matching fluid, and spectra are collected from angles between \pm 10 and $\pm 80^{\circ}$ at steps of $1^{\circ}$ with a liquid nitrogen-cooled CCD detector attached to a spectrometer (Acton SP2300i, PyLoN Princeton Instruments).

To analyze the angular-dependent spectra, we used an optical simulation together with a fitting procedure based on a dipole radiation model ${ }^{52}$ and a scattering matrix algorithm. ${ }^{53}$ The calculation takes microcavity effects and optical anisotropy into account, if necessary.

6.6. Transient Photoluminescence. To analyze the photoluminescence lifetime of the samples, we measured their decay upon excitation with a pulsed nitrogen laser (LTB MNL 200, wavelength $337 \mathrm{~nm}$, pulse width $<2 \mathrm{~ns}$ ) with a streak camera (Hamamatsu C5680) attached to a spectrograph (Acton SpectraPro 2300i Princeton Instruments). The aquired data was then averaged over the dominant part of the emission spectrum and a monoexponential curve was fitted to the data to obtain the phosphorescence lifetime.

6.7. Impedance Spectroscopy. For impedance spectroscopy, simple bilayer OLEDs with the stack layout consisting of $150 \mathrm{~nm}$ ITO, $30 \mathrm{~nm}$ PEDOT:PSS for hole injection, $70 \mathrm{~nm}$ NPB for hole transport, and $70 \mathrm{~nm}$ of DPEPO with different dopants, and finally 15 $\mathrm{nm}$ calcium topped with $100 \mathrm{~nm}$ of aluminum as a cathode were fabricated. In analogy to the usual stack design, the doped DPEPO is termed as the electron transport layer (ETL). If SOP with a negative charge density at the interface between HTL and ETL occurs, the HTL exhibits flat band conditions and hole injection at biases well below the built-in voltage $V_{\mathrm{bi}}$. This behavior leads to a characteristic step at the so-called threshold voltage $V_{\text {th }}$ in the capacitance-voltage measurement of the device, as clearly visible for the devices studied here (see Figure S6a).

The measurements were carried out with the Fluxim Paios measurement platform at frequencies between $13 \mathrm{~Hz}$ and $13 \mathrm{kHz}$ but only $133 \mathrm{~Hz}$ and $1.3 \mathrm{kHz}$ were used for the analysis, as parasitic effects reduce the data quality at higher and lower frequencies. The results were analyzed according to the literature. ${ }^{21,30}$

6.8. Kelvin Probe. Kelvin probe experiments were carried out with a MacAllister KP 6500 that is attached to a thermal deposition chamber. This allows for in situ measurement of the organic film's surface potential under high vacuum conditions without exposing the film to the ambient. Substrates with $150 \mathrm{~nm}$ ITO were used for film fabrication.

\section{ASSOCIATED CONTENT}

\section{Supporting Information}

The Supporting Information is available free of charge at https://pubs.acs.org/doi/10.1021/acsami.0c14613.

The PL spectra; moments of the ESP surface; the overview over PDMs and TDMs calculated with TDDFT; the results from ADPL; the graph correlating the magnitude of the PDM and the TDM orientation; the results from transient PL; exemplary capacitancevoltage characteristics; and Kelvin probe measurements (PDF)

\section{AUTHOR INFORMATION}

\section{Corresponding Author}

Wolfgang Brütting - Institute of Physics, University of Augsburg, 86135 Augsburg, Germany; ๑ orcid.org/00000001-9895-8281; Email: bruetting@physik.uniaugsburg.de

\section{Authors}

Markus Schmid - Institute of Physics, University of Augsburg, 86135 Augsburg, Germany

Kristoffer Harms - Applied Organic Materials, Institute for High Frequency Technology, Technical University of Braunschweig, 38106 Braunschweig, Germany

Carl Degitz - Institute of Nanotechnology, Karlsruhe Institute of Technology, 76344 Eggenstein-Leopoldshafen, Germany; Merck KGaA, Performance Materials - Display Solutions, 64293 Darmstadt, Germany

Thomas Morgenstern - Institute of Physics, University of Augsburg, 86135 Augsburg, Germany

Alexander Hofmann - Institute of Physics, University of Augsburg, 86135 Augsburg, Germany

Pascal Friederich - Institute of Theoretical Informatics and Institute of Nanotechnology, Karlsruhe Institute of Technology, 76131 Karlsruhe, Germany; 이이이.org/00000003-4465-1465

Hans-Hermann Johannes - Applied Organic Materials, Institute for High Frequency Technology, Technical University of Braunschweig, 38106 Braunschweig, Germany

Wolfgang Wenzel - Institute of Nanotechnology, Karlsruhe Institute of Technology, 76344 Eggenstein-Leopoldshafen, Germany 
Wolfgang Kowalsky - Applied Organic Materials, Institute for High Frequency Technology, Technical University of Braunschweig, 38106 Braunschweig, Germany

Complete contact information is available at: https://pubs.acs.org/10.1021/acsami.0c14613

\section{Notes}

The authors declare no competing financial interest.

\section{ACKNOWLEDGMENTS}

This work was funded by the German Research Foundation (DFG) under Contract No. BR 1728/20-1 and the German Ministry of Education and Research (BMBF) within the project "Interphase" under Contract Nos. 13N13664 and 13N13656. P.F. has received funding from the European Union's Horizon 2020 Research and Innovation Programme under the Marie Sklodowska-Curie Grant Agreement No. 795206.

\section{REFERENCES}

(1) Noguchi, Y.; Lim, H.; Isoshima, T.; Ito, E.; Hara, M.; Won Chin, W.; Wook Han, J.; Kinjo, H.; Ozawa, Y.; Nakayama, Y.; Ishii, H. Influence of the Direction of Spontaneous Orientation Polarization on the Charge Injection Properties of Organic Light-Emitting Diodes. Appl. Phys. Lett. 2013, 102, No. 203306.

(2) Altazin, S.; Züfle, S.; Knapp, E.; Kirsch, C.; Schmidt, T. D.; Jäger, L.; Noguchi, Y.; Brütting, W.; Ruhstaller, B. Simulation of OLEDs with a Polar Electron Transport Layer. Org. Electron. 2016, 39, 244249.

(3) Hofmann, A. J.; Züfle, S.; Shimizu, K.; Schmid, M.; Wessels, V.; Jäger, L.; Altazin, S.; Ikegami, K.; Khan, M. R.; Neher, D.; Ishii, H.; Ruhstaller, B.; Brütting, W. Dipolar Doping of Organic Semiconductors to Enhance Carrier Injection. Phys. Rev. Appl. 2019, 12, No. 064052.

(4) Yokoyama, D.; Sasabe, H.; Furukawa, Y.; Adachi, C.; Kido, J. Molecular Stacking Induced by Intermolecular C-H $\cdots \mathrm{N}$ Hydrogen Bonds Leading to High Carrier Mobility in Vacuum-Deposited Organic Films. Adv. Funct. Mater. 2011, 21, 1375-1382.

(5) Suzuki, K.; Kubo, S.; Aussenac, F.; Engelke, F.; Fukushima, T.; Kaji, H. Analysis of Molecular Orientation in Organic Semiconducting Thin Films Using Static Dynamic Nuclear Polarization Enhanced Solid-State NMR Spectroscopy. Angew. Chem., Int. Ed. 2017, 56, $14842-14846$.

(6) Frischeisen, J.; Yokoyama, D.; Adachi, C.; Brütting, W. Determination of Molecular Dipole Orientation in Doped Fluorescent Organic Thin Films by Photoluminescence Measurements. Appl. Phys. Lett. 2010, 96, No. 073302.

(7) Frischeisen, J.; Yokoyama, D.; Endo, A.; Adachi, C.; Brütting, W. Increased Light Outcoupling Efficiency in Dye-Doped Small Molecule Organic Light-Emitting Diodes with Horizontally Oriented Emitters. Org. Electron. 2011, 12, 809-817.

(8) Yokoyama, D. Molecular Orientation in Small-Molecule Organic Light-Emitting Diodes. J. Mater. Chem. 2011, 21, 19187.

(9) Brütting, W.; Frischeisen, J.; Schmidt, T. D.; Scholz, B. J.; Mayr, C. Device Efficiency of Organic Light-Emitting Diodes: Progress by Improved Light Outcoupling. Phys. Status Solidi A 2013, 210, 44-65.

(10) Schmidt, T. D.; Lampe, T.; Sylvinson, M. R. D.; Djurovich, P. I.; Thompson, M. E.; Brütting, W. Emitter Orientation as a Key Parameter in Organic Light-Emitting Diodes. Phys. Rev. Appl. 2017, 8, No. 037001.

(11) Callens, M. K.; Yokoyama, D.; Neyts, K. Anisotropic Materials in OLEDs for High Outcoupling Efficiency. Opt. Express 2015, 23, 21128-21148.

(12) Schmid, M.; Morgenstern, T.; Brütting, W. Enabling Electron Conduction in Anisotropic Hole Transport Materials for Superior
Optical Properties in Organic Light Emitting Diodes. Org. Electron. 2018, 62, 216-219.

(13) Flämmich, M.; Frischeisen, J.; Setz, D. S.; Michaelis, D.; Krummacher, B. C.; Schmidt, T. D.; Brütting, W.; Danz, N. Oriented Phosphorescent Emitters Boost OLED Efficiency. Org. Electron. 2011, $12,1663-1668$.

(14) Kim, K.-H.; Kim, J.-J. Origin and Control of Orientation of Phosphorescent and TADF Dyes for High-Efficiency OLEDs. Adv. Mater. 2018, 30, No. 1705600.

(15) Kim, S.-Y.; Jeong, W.-I.; Mayr, C.; Park, Y.-S.; Kim, K.-H.; Lee, J.-H.; Moon, C.-K.; Brütting, W.; Kim, J.-J. Organic Light-Emitting Diodes with $30 \%$ External Quantum Efficiency Based on a Horizontally Oriented Emitter. Adv. Funct. Mater. 2013, 23, 38963900.

(16) Kim, K.-H.; Lee, S.; Moon, C.-K.; Kim, S.-Y.; Park, Y.-S.; Lee, J.-H.; Woo Lee, J.; Huh, J.; You, Y.; Kim, J.-J. Phosphorescent DyeBased Supramolecules for High-Efficiency Organic Light-Emitting Diodes. Nat. Commun. 2014, 5, No. 4769.

(17) Kim, K.-H.; Ahn, E. S.; Huh, J.-S.; Kim, Y.-H.; Kim, J.-J. Design of Heteroleptic Ir Complexes with Horizontal Emitting Dipoles for Highly Efficient Organic Light-Emitting Diodes with an External Quantum Efficiency of 38\%. Chem. Mater. 2016, 28, 7505-7510.

(18) Friederich, P.; Coehoorn, R.; Wenzel, W. Molecular Origin of the Anisotropic Dye Orientation in Emissive Layers of Organic Light Emitting Diodes. Chem. Mater. 2017, 29, 9528-9535.

(19) Moon, C.-K.; Kim, K.-H.; Kim, J.-J. Unraveling the Orientation of Phosphors Doped in Organic Semiconducting Layers. Nat. Commun. 2017, 8, No. 685.

(20) Tonnelé, C.; Stroet, M.; Caron, B.; Clulow, A. J.; Nagiri, R. C. R.; Malde, A. K.; Burn, P. L.; Gentle, I. R.; Mark, A. E.; Powell, B. J. Elucidating the Spatial Arrangement of Emitter Molecules in Organic Light-Emitting Diode Films. Angew. Chem., Int. Ed. 2017, 56, 84028406.

(21) Morgenstern, T.; Schmid, M.; Hofmann, A.; Bierling, M.; Jäger, L.; Brütting, W. Correlating Optical and Electrical Dipole Moments to Pinpoint Phosphorescent Dye Alignment in Organic Light-Emitting Diodes. ACS Appl. Mater. Interfaces 2018, 10, 31541-31551.

(22) Graf, A.; Liehm, P.; Murawski, C.; Hofmann, S.; Leo, K.; Gather, M. C. Correlating the Transition Dipole Moment Orientation of Phosphorescent Emitter Molecules in OLEDs with Basic Material Properties. J. Mater. Chem. C 2014, 2, 10298-10304.

(23) Jurow, M. J.; Mayr, C.; Schmidt, T. D.; Lampe, T.; Djurovich, P. I.; Brütting, W.; Thompson, M. E. Understanding and Predicting the Orientation of Heteroleptic Phosphors in Organic Light-Emitting Materials. Nat. Mater. 2016, 15, 85-91.

(24) Tamayo, A. B.; Alleyne, B. D.; Djurovich, P. I.; Lamansky, S.; Tsyba, I.; Ho, N. N.; Bau, R.; Thompson, M. E. Synthesis and Characterization of Facial and Meridional Tris-Cyclometalated Iridium(III) Complexes. J. Am. Chem. Soc. 2003, 125, 7377-7387.

(25) Ide, N.; Matsusue, N.; Kobayashi, T.; Naito, H. Photoluminescence Properties of Facial- and Meridional-Ir(ppy)3 Thin Films. Thin Solid Films 2006, 509, 164-167.

(26) Harms, K. Molekulare Orientierung von Halbleitermaterialien in Dünnschichtsystemen. Dissertation; Technical University CaroloWilhelmina: Braunschweig, 2020.

(27) Schrödinger Release 2019-4: Materials Science Suite; Schrodinger, LLC: New York, NY, 2019.

(28) Mori, K.; Goumans, T. P. M.; van Lenthe, E.; Wang, F. Predicting Phosphorescent Lifetimes and Zero-Field Splitting of Organometallic Complexes with Time-Dependent Density Functional Theory Including Spin-Orbit Coupling. Phys. Chem. Chem. Phys. 2014, 16, 14523-14530.

(29) Neumann, T.; Danilov, D.; Lennartz, C.; Wenzel, W. Modeling Disordered Morphologies in Organic Semiconductors. J. Comput. Chem. 2013, 34, 2716-2725.

(30) Jäger, L.; Schmidt, T. D.; Brütting, W. Manipulation and Control of the Interfacial Polarization in Organic Light-Emitting Diodes by Dipolar Doping. AIP Adv. 2016, 6, No. 095220. 
(31) Berleb, S.; Brütting, W.; Paasch, G. Interfacial Charges and Electric Field Distribution in Organic Hetero-Layer Light-Emitting Devices. Org. Electron. 2000, 1, 41-47.

(32) Nowy, S.; Ren, W.; Elschner, A.; Lövenich, W.; Brütting, W. Impedance Spectroscopy as a Probe for the Degradation of Organic Light-Emitting Diodes. J. Appl. Phys. 2010, 107, No. 054501.

(33) Friederich, P.; Rodin, V.; von Wrochem, F.; Wenzel, W. BuiltIn Potentials Induced by Molecular Order in Amorphous Organic Thin Films. ACS Appl. Mater. Interfaces 2018, 10, 1881-1887.

(34) Noguchi, Y.; Brütting, W.; Ishii, H. Spontaneous orientation Polarization in Organic Light-Emitting Diodes. Jpn. J. Appl. Phys. 2019, 58, No. SF0801.

(35) Osada, K.; Goushi, K.; Kaji, H.; Adachi, C.; Ishii, H.; Noguchi, Y. Observation of Spontaneous Orientation Polarization in Evaporated Films of Organic Light-Emitting Diode Materials. Org. Electron. 2018, 58, 313-317.

(36) Noguchi, Y.; Osada, K.; Ninomiya, K.; Gunawardana, H. D.; Koswattage, K. R.; Ishii, H. Influence of Intermolecular Interactions on the Formation of Spontaneous Orientation Polarization in Organic Semiconducting Films. J. Soc. Inf. Disp. 2020, 1-9, DOI: 10.1002/ jsid.956.

(37) Kim, K.-H.; Yoo, S.-J.; Kim, J.-J. Boosting Triplet Harvest by Reducing Nonradiative Transition of Exciplex toward Fluorescent Organic Light-Emitting Diodes with 100\% Internal Quantum Efficiency. Chem. Mater. 2016, 28, 1936-1941.

(38) Moon, C.-K.; Kim, K.-H.; Lee, J. W.; Kim, J.-J. Influence of Host Molecules on Emitting Dipole Orientation of Phosphorescent Iridium Complexes. Chem. Mater. 2015, 27, 2767-2769.

(39) Lampe, T.; Schmidt, T. D.; Jurow, M. J.; Djurovich, P. I.; Thompson, M. E.; Brütting, W. Dependence of Phosphorescent Emitter Orientation on Deposition Technique in Doped Organic Films. Chem. Mater. 2016, 28, 712-715.

(40) Naqvi, B. A.; Schmid, M.; Crovini, E.; Sahay, P.; Naujoks, T.; Rodella, F.; Zhang, Z.; Strohriegl, P.; Bräse, S.; Zysman-Colman, E.; Brütting, W. What Controls the Orientation of TADF Emitters? Front. Chem. 2020, 8, No. 060101.

(41) Miyata, K.; Ohba, T.; Kobayashi, A.; Kato, M.; Nakanishi, T.; Fushimi, K.; Hasegawa, Y. Thermostable Organo-phosphor: LowVibrational Coordination Polymers That Exhibit Different Intermolecular Interactions. ChemPlusChem 2012, 77, 277-280.

(42) Pritchard, B. P.; Altarawy, D.; Didier, B.; Gibson, T. D.; Windus, T. L. New Basis Set Exchange: An Open, Up-to-Date Resource for the Molecular Sciences Community. J. Chem. Inf. Model. 2019, 59, 4814-4820.

(43) Frisch, M. J.; Trucks, G. W.; Schlegel, H. B.; Scuseria, G. E.; Robb, M. A.; Cheeseman, J. R.; Sclamani, G.; Barone, V.; Mennucci, B.; Petersson, G. A.; Nakatsuji, H.; Caricato, M.; Li, X.; Hratchina, H. P.; Izmaylov, A. F.; Bloino, J.; Zheng, G.; Sonnenberg, J. L.; Hada, M.; Ehara, M.; Toyota, K.; Fukuda, R.; Hasegawa, J.; Ishida, M.; Honda, Y.; Kitao, O.; Nakai, H.; Vreven, T.; Montgomery, J. A.; Peralta, J. E.; Ogliaro, F.; Bearpark, M.; Heyd, J. J.; Brothers, E.; Kudin, K. N.; Staroverov, V. N.; Kobayashi, R.; Normand, J.; Raghavachari, K.; Rendell, A.; Burant, J. C.; Iyengar, S. S.; Tomasi, J.; Cossi, M.; Rega, N.; Millam, J. M.; Klene, M.; Knox, J. E.; Bakken, V.; Adamo, C.; Jaramillo, J.; Gomperts, R.; Stratman, R. E.; Yazyev, O.; Austin, A. J.; Cammi, R.; Pomelli, C.; Ochterski, J. W.; Martin, R. L.; Morokuma, K.; Zakrzewski, V. G.; Voth, G. A.; Salvador, P.; Dannenberg, J. J.; Dapprich, S.; Daniels, A. D.; Farkas, O.; Foresman, J. B.; Ortiz, J. V.; Cioslowski, J.; Fox, D. J. Gaussian 09, revision A.02; Gaussian Inc.: Wallingford, CT, 2009.

(44) TURBOMOLE V7.4 2019, A Development of University of Karlsruhe and Forschungszentrum Karlsruhe GmbH, 1989-2007; TURBOMOLE GmbH, 2019. www.turbomole.com.

(45) Balasubramani, S. G.; Chen, G. P.; Coriani, S.; Diedenhofen, M.; Frank, M. S.; Franzke, Y. J.; Furche, F.; Grotjahn, R.; Harding, M. E.; Hättig, C.; Hellweg, A.; Helmich-Paris, B.; Holzer, C.; Huniar, U.; Kaupp, M.; Marefat Khah, A.; Karbalaei Khani, S.; Müller, T.; Mack, F.; Nguyen, B. D.; Parker, S. M.; Perlt, E.; Rappoport, D.; Reiter, K.; Roy, S.; Rückert, M.; Schmitz, G.; Sierka, M.; Tapavicza, E.; Tew, D.
P.; van Wüllen, C.; Voora, V. K.; Weigend, F.; Wodyński, A.; Yu, J. M. TURBOMOLE: Modular Program Suite for Ab Initio QuantumChemical and Condensed-Matter Simulations. J. Chem. Phys. 2020, 152, No. 184107.

(46) Andrae, D.; Huermann, U.; Dolg, M.; Stoll, H.; Preu, H. Energy-Adjusted Ab Initio Pseudopotentials for the Second and Third Row Transition Elements. Theor. Chim. Acta 1990, 77, 123-141.

(47) Aidas, K.; Angeli, C.; Bak, K. L.; Bakken, V.; Bast, R.; Boman; Christiansen, O.; Cimiraglia, R.; Coriani, S.; Dahle, P.; Dalskov, E. K.; Ekström, U.; Enevoldsen, T.; Eriksen, J. J.; Ettenhuber, P.; Fernández, B.; Ferrighi, L.; Fliegl, H.; Frediani, L.; Hald, K.; Halkier, A.; Hättig, C.; Heiberg, H.; Helgaker, T.; Hennum, A. C.; Hettema, H.; Hjertenæs, E.; Host, S.; Hoyvik, I.-M.; Iozzi, M. F.; Jansik, B.; Jensen, H.; Jonsson, D.; Jorgensen, P.; Kauczor, J.; Kirpekar, S.; Kjaergaard, T.; Klopper, W.; Knecht, S.; Kobayashi, R.; Koch, H.; Kongsted, J.; Krapp, A.; Kristensen, K.; Ligabue, A.; Lutnaes, O. B.; Melo, J. I.; Mikkelsen, K. V.; Myhre, R. H.; Neiss, C.; Nielsen, C. B.; Norman, P.; Olsen, J.; Olsen, J. M. H.; Osted, A.; Packer, M. J.; Pawlowski, F.; Pedersen, T. B.; Provasi, P. F.; Reine, S.; Rinkevicius, Z.; Ruden, T. A.; Ruud, K.; Rybkin, V. V.; Sałek, P.; Samson, C. C. M.; de Merás, A. S.; Saue, T.; Sauer, S. P. A.; Schimmelpfennig, B.; Sneskov, K.; Steindal, A. H.; Sylvester-Hvid, K. O.; Taylor, P. R.; Teale, A. M.; Tellgren, E. I.; Tew, D. P.; Thorvaldsen, A. J.; Thogersen, L.; Vahtras, O.; Watson, M. A.; Wilson, D. J. D.; Ziolkowski, M.; Agren, H. The Dalton Quantum Chemistry Program System. WIREs Comput. Mol. Sci. 2014, 4, 269-284.

(48) Dalton: A Molecular Electronic Structure Program, Release v2018.0; (November 19, 2018) https://daltonprogram.org/.

(49) Koseki, S.; Schmidt, M. W.; Gordon, M. S. MCSCF/6$31 \mathrm{G}(\mathrm{d}, \mathrm{p})$ Calculations of One-Electron Spin-Orbit Coupling Constants in Diatomic Molecules. J. Phys. Chem. A. 1992, 96, 10768-10772.

(50) Koseki, S.; Gordon, M. S.; Schmidt, M. W.; Matsunaga, N. Main Group Effective Nuclear Charges for Spin-Orbit Calculations. J. Phys. Chem. B. 1995, 99, 12764-12772.

(51) Koseki, S.; Schmidt, M. W.; Gordon, M. S. Effective Nuclear Charges for the First- through Third-Row Transition Metal Elements in Spin-Orbit Calculations. J. Phys. Chem. A 1998, 102, 10430-10435.

(52) Penninck, L.; de Visschere, P.; Beeckman, J.; Neyts, K. Dipole Radiation Within One-Dimensional Anisotropic Microcavities: A Simulation Method. Opt. Express 2011, 19, 18558-18576.

(53) Ko, D. Y. K.; Sambles, J. R. Scattering Matrix Method for Propagation of Radiation in Stratified Media: Attenuated Total Reflection Studies of Liquid Crystals. J. Opt. Soc. Am. A 1988, 5, 1863. 